\title{
Alternative wetting boundary condition for the chemical-potential-based free-energy lattice Boltzmann model
}

\author{
Y. Yu, Q. Li, ${ }^{*}$ and R. Z. Huang \\ School of Energy Science and Engineering, Central South University, Changsha 410083, China
}

(Received 16 December 2020; revised 8 June 2021; accepted 15 June 2021; published 8 July 2021)

\begin{abstract}
The free-energy lattice Boltzmann (LB) method is a multiphase LB approach based on the thermodynamic theory. Compared with traditional free-energy LB models, which employ a nonideal thermodynamic pressure tensor, the chemical-potential-based free-energy LB model has attracted much attention in recent years as it avoids computing the thermodynamic pressure tensor and its divergence. In this paper, we propose an improved wetting boundary condition for the chemical-potential-based free-energy LB model. Different from the original wetting boundary condition in the literature, the improved wetting boundary condition utilizes a surface chemical potential that is compatible with the chemical potential of the fluid domain. Accordingly, the thermodynamic consistency of the chemical-potential-based free-energy LB model can be retained by the improved wetting boundary condition. Numerical simulations are performed for droplets resting on flat and cylindrical surfaces with different contact angles. The numerical results show that the improved wetting boundary condition yields more reasonable results and the maximum spurious velocities are found to be smaller by $2 \sim 3$ orders of magnitude than those produced by the original wetting boundary condition.
\end{abstract}

DOI: 10.1103/PhysRevE.104.015303

\section{INTRODUCTION}

Understanding multiphase flows is very important for a wide range of industrial applications [1], such as oil recovery and subsurface storage of carbon dioxide. However, the dynamics of interfaces involved in multiphase flows is difficult to study since the interfaces between different phases are usually deformable and their shapes are not known a priori. To capture an interface or track the motion of an interface, many numerical methods have been developed, such as the front-tracking method [2], the volume of fluid (VOF) method [3], and the level set method [4]. The front-tracking method is usually unable to simulate the interface coalescence or breakup [1], while in the VOF and level set methods the interface reconstruction step or interface reinitialization is required [5]. Moreover, numerical instability may appear when the VOF and level set methods are employed to simulate surface-tension-dominated flows in complex geometries such as porous media.

In the past three decades, the lattice Boltzmann (LB) method has been developed into an efficient numerical method for simulating fluid flow and heat transfer [6-11]. Owing to its mesoscopic features, the LB method has shown some distinctive advantages over conventional numerical methods and has been widely used to simulate multiphase flows and interfacial phenomena. The existing multiphase LB models can be generally classified into four categories, i.e., the pseudopotential

${ }^{*}$ Corresponding author: qingli@csu.edu.cn

Published by the American Physical Society under the terms of the Creative Commons Attribution 4.0 International license. Further distribution of this work must maintain attribution to the author(s) and the published article's title, journal citation, and DOI.
LB model [12-20], the color-gradient LB model [21-23], the free-energy LB model [24,25], and the phase-field LB model [26-28]. Among these multiphase LB models, the free-energy model proposed by Swift et al. [24,25] was devised based on the thermodynamic theory, which starts with a free-energy functional that contains the thermodynamics of the intended system, and then other relevant physical quantities can be derived from the free-energy functional [29].

In the original free-energy LB model [24,25], the thermodynamics of a multiphase system is incorporated into the model by modifying the second-order moment of the equilibrium density distribution function to include a nonideal thermodynamic pressure tensor. Nevertheless, the original free-energy LB model was found to break the Galilean invariance owing to some non-Navier-Stokes terms recovered in the momentum equation [30]. In the literature there are mainly two approaches that can be used to overcome the drawback of the original free-energy LB model: one is to add some correction terms to the equilibrium density distribution function $[25,31-33]$ so as to restore the Galilean invariance, and the other is using a forcing term to incorporate the thermodynamics of a multiphase system into the LB equation, which was proposed by Wagner and Li [34]. In their free-energy LB model, a forcing term is employed to incorporate the divergence of a nonideal pressure tensor into the LB equation. A similar LB model can be found in the study of Lee and Fischer [35], in which the divergence of the nonideal pressure tensor is expressed by its chemical-potential form. This class of free-energy LB models has attracted much attention in recent years [36,37].

When the multiphase modeling involves a solid wall, it is necessary to implement a wetting boundary condition that accounts for the contact angle between the fluid interface and the solid wall. The first study of wetting phenomena using 
the free-energy LB model is attributed to Briant et al. [38]. They proposed a contact angle scheme to implement the wettability of a solid surface in the free-energy LB simulations. Their scheme has been extensively used in investigating wetting phenomena by the free-energy LB model. For example, Dupuis and Yeomans [39] employed the scheme of Briant et al. [38] to study the spreading of droplets on chemically heterogeneous surfaces. Nevertheless, it should be noted that in the scheme of Briant et al. [38] the free-energy density was chosen to be a special form (other than the van der Waals free-energy density). The choice of Briant et al. brings an advantage that the wall-fluid surface tensions can be calculated in a closed form, but it prevents the incorporation of traditional nonideal equations of state into the free-energy LB model, such as the van der Waals equation of state and the Peng-Robinson equation of state [40].

The fluid-solid interaction scheme, which is a well-known contact angle scheme for the pseudopotential multiphase LB model, has also been introduced into the free-energy LB model by Mazloomi M. et al. [41]. In their study, the PengRobinson equation of state is utilized and the multiphase thermodynamics is incorporated via a nonideal force given by $\mathbf{F}=\nabla\left(\rho c_{\mathrm{s}}^{2}\right)-\nabla \cdot \mathbf{P}$, where $\rho$ is the density, $c_{\mathrm{s}}$ is the lattice sound speed, and $\mathbf{P}$ is the nonideal pressure tensor. Recently, Wen et al. [42] proposed a wetting boundary condition for the chemical-potential-based free-energy LB model, in which the nonideal force is expressed by $\mathbf{F}=\boldsymbol{\nabla}\left(\rho c_{\mathrm{s}}^{2}\right)-\rho \nabla \mu_{c}$, where $\mu_{c}$ is the chemical potential. A surface chemical potential was used at the solid surface and different contact angles were simulated by adjusting the value of the surface chemical potential. Such a wetting boundary condition is easy to implement but the study of Wen et al. [42] shows that it leads to very large spurious velocities (on the order of $0.01 \sim 0.05$ ). Besides, it should be noted that tuning the surface chemical potential over a wide range to achieve different contact angles is seemingly inconsistent with the thermodynamic theory.

The aim of the present work is therefore to develop an improved wetting boundary condition for the chemicalpotential-based free-energy LB model. In comparison with the original wetting boundary condition devised in Ref. [42], the improved wetting boundary condition employs a surface chemical potential that is compatible with the chemical potential of the fluid domain and can significantly reduce the spurious velocities. The rest of the present paper is organized as follows. The chemical-potential-based free-energy LB model is briefly introduced in Sec. II. The improved wetting boundary condition is presented in Sec. III. Numerical simulations are carried out in Sec. IV. A brief summary is finally given in Sec. V.

\section{CHEMICAL-POTENTIAL-BASED FREE-ENERGY LB MODEL}

In this study, a two-dimensional (2D) nine-velocity (D2Q9) LB model with a multiple-relaxation-time (MRT) collision operator $[43,44]$ is employed. Generally, the MRT-LB equation can be written as follows $[45,46]$ :

$$
\begin{aligned}
f_{\alpha}\left(\mathbf{x}+\mathbf{e}_{\alpha} \delta_{t}, t+\delta_{t}\right)= & f_{\alpha}(\mathbf{x}, t)-\left.\Lambda_{\alpha \beta}\left(f_{\beta}-f_{\beta}^{\mathrm{eq}}\right)\right|_{(\mathbf{x}, t)} \\
& +\left.\delta_{t}\left(G_{\alpha}-0.5 \Lambda_{\alpha \beta} G_{\beta}\right)\right|_{(\mathbf{x}, t)},
\end{aligned}
$$

where $f_{\alpha}$ is the density distribution function, $f_{\alpha}^{\text {eq }}$ is the equilibrium density distribution function, $\mathbf{x}$ is the spatial position, $t$ is the time, $\delta_{t}$ is the time step, $\mathbf{e}_{\alpha}$ is the discrete velocity in the $\alpha$ th direction, $G_{\alpha}$ is the forcing term in the discrete velocity space, and $\Lambda_{\alpha \beta}=\left(\mathbf{M}^{-1} \bar{\Lambda} \mathbf{M}\right)_{\alpha \beta}$ is the collision operator, in which $\mathbf{M}$ is a transformation matrix and $\overline{\mathbf{\Lambda}}$ is a diagonal matrix $[45,46]$. For the D2Q9 lattice, the diagonal matrix is given by $\overline{\mathbf{\Lambda}}=\operatorname{diag}\left(\tau_{c}^{-1}, \tau_{e}^{-1}, \tau_{\varsigma}^{-1}, \tau_{c}^{-1}, \tau_{q}^{-1}, \tau_{c}^{-1}, \tau_{q}^{-1}, \tau_{v}^{-1}, \tau_{v}^{-1}\right)$,

where $\tau_{c}=1$ is the nondimensional relaxation time related the conserved moments; $\tau_{\zeta}$ and $\tau_{q}$ are free parameters; while $\tau_{e}$ and $\tau_{v}$ are the nondimensional relaxation times determining the bulk and shear viscosities, respectively.

Through the transformation matrix $\mathbf{M}$, the right-hand side of Eq. (1) can be implemented in the moment space:

$$
\mathbf{m}^{*}=\mathbf{m}-\overline{\mathbf{\Lambda}}\left(\mathbf{m}-\mathbf{m}^{\mathrm{eq}}\right)+\delta_{t}\left(\mathbf{I}-\frac{\bar{\Lambda}}{2}\right) \mathbf{S}
$$

where $\mathbf{m}=\mathbf{M f}, \mathbf{m}^{\mathrm{eq}}=\mathbf{M f} \mathbf{f}^{\mathrm{eq}}$, and $\mathbf{S}=\mathbf{M G}$ is the forcing term in the moment space. Then the streaming step can be implemented as follows:

$$
f_{\alpha}\left(\mathbf{x}+\mathbf{e}_{\alpha} \delta_{t}, t+\delta_{t}\right)=f_{\alpha}^{*}(\mathbf{x}, t),
$$

where $\mathbf{f}^{*}=\mathbf{M}^{-1} \mathbf{m}^{*}$ and $\mathbf{M}^{-1}$ is the inverse matrix of the transformation matrix. The macroscopic density and velocity can be calculated by

$$
\rho=\sum_{\alpha} f_{\alpha}, \quad \rho \mathbf{u}=\sum_{\alpha} \mathbf{e}_{\alpha} f_{\alpha}+\frac{\delta_{t}}{2} \mathbf{F},
$$

where $\mathbf{F}$ is the force exerted on the system. For the standard chemical-potential-based free-energy LB model, the nonideal force is given by $[35,42]$

$$
\mathbf{F}=\nabla\left(\rho c_{s}^{2}\right)-\rho \nabla \mu_{c},
$$

where the chemical potential $\mu_{c}$ is defined as the variation of the free energy functional $\mathcal{F}$ with respect to the density, i.e.,

$$
\mu_{c}=\frac{\delta \mathcal{F}}{\delta \rho}=E_{f}^{\prime}(\rho)-\kappa \nabla^{2} \rho,
$$

where $\kappa$ is a positive constant and $E_{f}(\rho)$ represents the bulk free-energy density, which leads to an equation of state that allows for the coexistence of liquid and gas phases.

The standard chemical-potential-based free-energy LB model was found to suffer from the problem of thermodynamic inconsistency due to the force imbalance at the discrete lattice level [47]. Several schemes have been proposed in the literature to eliminate the thermodynamic inconsistency of the chemical-potential-based free-energy LB model, such as the forcing scheme devised by Wagner [48], the Lax-Wendroff propagation scheme formulated by Lou and Guo [48], and an improved scheme proposed by Li et al. [49] based on the MRT collision operator. In the present study we adopt the improved scheme developed by Li et al. [49] to eliminate the thermodynamic inconsistency of the chemical-potential-based free-energy LB model.

For the van der Waals equation of state [24,25,42], the bulk free-energy density is given by $E_{f}(\rho)=$ 


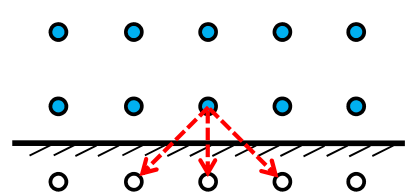

solid nodes

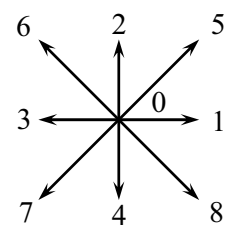

FIG. 1. Sketch of solid nodes beneath a straight wall.

$\rho R T \ln [\rho /(1-b \rho)]-a \rho^{2}$, which corresponds to the following chemical potential:

$$
\mu_{c}=R T\left[\ln \left(\frac{\rho}{1-b \rho}\right)+\frac{1}{1-b \rho}\right]-2 a \rho-\kappa \nabla^{2} \rho,
$$

where $a$ is the attraction parameter, $b$ is the repulsion parameter, and $R$ is the gas constant. In numerical simulations, the gradient terms are evaluated by the following isotropic finite-difference scheme:

$$
\nabla \phi=\frac{1}{c_{s}^{2} \delta_{t}} \sum_{\alpha} \omega_{\alpha} \phi\left(\mathbf{x}+\mathbf{e}_{\alpha} \delta_{t}\right) \mathbf{e}_{\alpha},
$$

where the weights $\omega_{\alpha}$ are given by $\omega_{0}=4 / 9, \omega_{1-4}=1 / 9$, and $\omega_{5-8}=1 / 36$.

\section{IMPROVED WETTING BOUNDARY CONDITION}

As previously mentioned, the first study of wetting phenomena using the free-energy LB model was conducted by Briant et al. [38]. In their work, the free-energy density was designed to be a special form, which leads to a special equation of state. Therefore the contact angle scheme formulated by Briant et al. [38] is not suitable for the free-energy LB model using classic nonideal equations of state. Recently, Wen et al. [42] proposed a chemical-potential boundary condition to implement the wettability of a solid surface for the chemical-potential-based free-energy LB model. In their wetting boundary condition, a surface chemical potential is introduced [42]:

$$
\mu_{c}\left(\mathbf{x}_{s}\right)=C,
$$

where $\mathbf{x}_{s}$ denotes a solid node (see Fig. 1) and $C$ is a constant. Meanwhile, the density of the solid node $\rho\left(\mathbf{x}_{s}\right)$ is determined according to its neighboring fluid nodes. The value of the surface chemical potential is adjusted to tune the contact angle. For the cases shown in Ref. [42], the constant $C$ in Eq. (10) is changed from $C=-0.23$ for $\theta \approx 30^{\circ}$ to $C=0.18$ for $\theta \approx 180^{\circ}$.

However, the aforementioned wetting boundary condition was found to yield very large spurious velocities [42]. Moreover, the thermodynamic theory tells us that at the thermodynamically equilibrium state the chemical potential is constant everywhere for a two-phase system with a fixed temperature. In other words, tuning the surface chemical potential over a wide range may be inconsistent with the thermodynamic theory. Hence we propose to use the following surface chemical potential:

$$
\mu_{c}\left(\mathbf{x}_{s}\right)=\frac{\sum_{\alpha} \omega_{\alpha} \mu_{c}\left(\mathbf{x}_{s}+\mathbf{e}_{\alpha} \delta_{t}\right) s_{w}}{\sum_{\alpha} \omega_{\alpha} s_{w}},
$$

where $\mathbf{x}_{s}+\mathbf{e}_{\alpha} \delta_{t}$ denotes the neighboring nodes of the solid node and the switch function $s_{w}$ equals 1 when $\mathbf{x}_{s}+\mathbf{e}_{\alpha} \delta_{t}$ is a fluid node but is zero when it is a solid node. With the above treatment, the surface chemical potential is compatible with the chemical potential of the fluid domain.

In a similar way, we can obtain an average density for each solid node, i.e.,

$$
\rho_{\mathrm{ave}}\left(\mathbf{x}_{s}\right)=\frac{\sum_{\alpha} \omega_{\alpha} \rho\left(\mathbf{x}_{s}+\mathbf{e}_{\alpha} \delta_{t}\right) s_{w}}{\sum_{\alpha} \omega_{\alpha} s_{w}} .
$$

Using this average density, the boundary nodes can be classified into three regions: the liquid-phase region, the gas-phase region, and the region of three-phase contact lines, where the fluid interface meets the solid boundary. In our simulations, we utilize $0.05 \rho_{l}+0.95 \rho_{g} \leqslant \rho_{\text {ave }}\left(\mathbf{x}_{s}\right) \leqslant 0.95 \rho_{l}+0.05 \rho_{g}$ to identify the region of three-phase contact lines, in which $\rho_{l}$ and $\rho_{g}$ are the densities of the liquid and gas phases, respectively. For each single-phase region, the density of a solid node is directly set to be the average density given by Eq. (12), i.e., $\rho\left(\mathbf{x}_{s}\right)=\rho_{\mathrm{ave}}\left(\mathbf{x}_{s}\right)$. For the region of three-phase contact lines, the solid node density is determined as follows:

$$
\rho\left(\mathbf{x}_{s}\right)= \begin{cases}\varphi \rho_{\mathrm{ave}}\left(\mathbf{x}_{s}\right), & \text { for } \theta \leqslant \theta_{s}, \\ \rho_{\mathrm{ave}}\left(\mathbf{x}_{s}\right)-\Delta \rho, & \text { for } \theta>\theta_{s},\end{cases}
$$

where $\varphi \geqslant 1$ and $\Delta \rho>0$ are constants employed to adjust the contact angle. The contact angle $\theta_{s} \approx 90^{\circ}$ corresponds to the standard case $\rho\left(\mathbf{x}_{s}\right)=\rho_{\text {ave }}\left(\mathbf{x}_{s}\right)$. Such a treatment is inspired by the study of Li et al. [50]. A limiter should be applied to Eq. (13), i.e., the solid node density given by Eq. (13) is constrained within $\left[0.05 \rho_{l}+0.95 \rho_{g}, 0.95 \rho_{l}+0.05 \rho_{g}\right]$. Here it should be emphasized that for all the solid nodes the surface chemical potential is always calculated by Eq. (11).

In addition, we would like to make a comparison between the present wetting boundary condition and the geometric formulation scheme [51,52]. The advantage of the geometric formulation scheme lies in that it can give a slope of the liquidgas interface that is basically consistent with a prescribed value of the contact angle. However, the numerical implementation of this scheme for 2D and 3D curved surfaces is very complicated, which involves a great number of interpolations, e.g., its implementation for $2 \mathrm{D}$ curved surfaces can be found

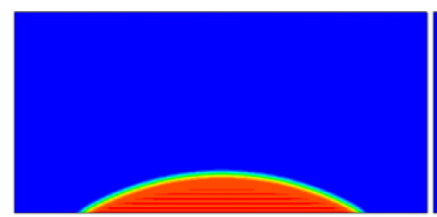

(a)

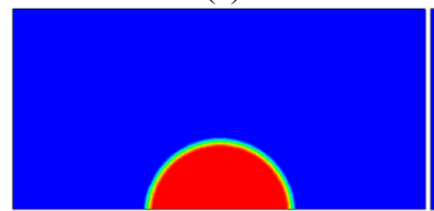

(c)

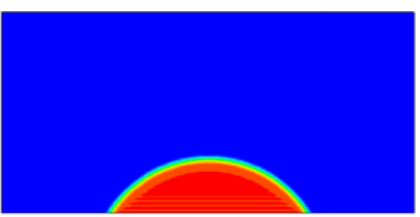

(b)

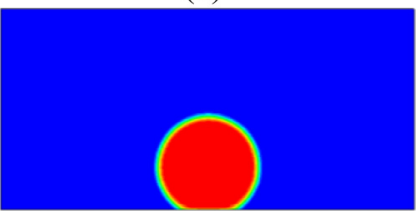

(d)
FIG. 2. Contact angles simulated by the original wetting boundary condition [42]. (a) $\mu_{c}=-0.065, \theta \approx 34^{\circ}$, (b) $\mu_{c}=-0.047$, $\theta \approx 61^{\circ}$, (c) $\mu_{c}=-0.01, \theta \approx 90^{\circ}$, and (d) $\mu_{c}=0.112, \theta \approx 152^{\circ}$. 


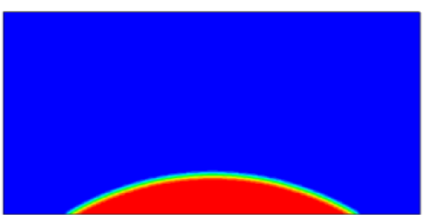

(a)

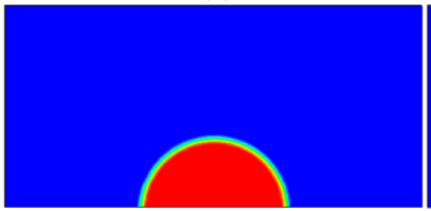

(c)

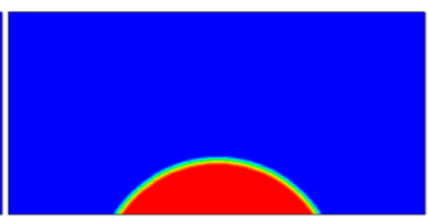

(b)

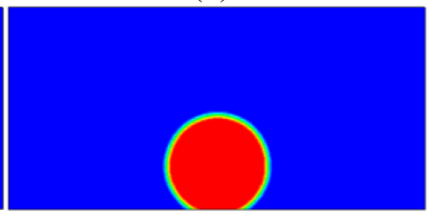

(d)
FIG. 3. Contact angles simulated by the improved wetting boundary condition. (a) $\varphi=1.6, \theta \approx 34^{\circ}$, (b) $\varphi=1.3, \theta \approx 61^{\circ}$, (c) $\varphi=1.02, \theta \approx 90^{\circ}$, and (d) $\Delta \rho=1.8, \theta \approx 152^{\circ}$.

in Ref. [52] (see Figs. 4 and 5 in the reference and the related descriptions therein).

\section{NUMERICAL SIMULATIONS}

In the previous section, an improved wetting boundary condition has been devised for the chemical-potential-based free-energy LB model. In this section numerical simulations are carried out to validate the proposed wetting boundary condition.

\section{A. Droplet resting on a flat surface}

The test of a droplet resting on a flat surface is first considered to verify the capability of the improved wetting boundary condition for simulating different contact angles on a flat surface. The computational domain is taken as $L_{x} \times L_{y}=180$ l.u. $\times 90$ l.u., in which 1.u. represents lattice units. The flat surface is located at the bottom of the computational domain. Initially, a semicircular droplet is placed at the center of the flat surface. The initial radius of the droplet is 30 l.u. In simulations, the parameters in Eq. (8) are chosen as follows: $a=9 / 392, b=2 / 21, R=1$, and $\kappa=0.02$. The critical density and temperature are given by $\rho_{c}=1 /(3 b)=3.5$ and $T_{c}=8 a /(27 R b)=1 / 14$, respectively. Our simulations are conducted at $T=0.7 T_{c}$, which corresponds to the coexisting densities $\rho_{l} \approx 7.52$ and $\rho_{g} \approx 0.46$ for circular droplets. The kinematic viscosity is chosen as $v=0.15$. The periodic boundary condition is applied in the horizontal direction, whereas the halfway bounce-back scheme is employed at the bottom and top boundaries. The wetting boundary condition is imposed at the flat surface.

The simulation results obtained by the original wetting boundary condition [42] and the improved wetting boundary condition at $t=6 \times 10^{4} \delta_{t}$ are shown in Figs. 2 and 3, respectively. Both types of the wetting boundary conditions are shown to be capable of modeling contact angles over a wide range. Nevertheless, the two wetting boundary conditions are found to be different in the following aspects. First, they exhibit different performances in terms of the thermodynamic consistency. To illustrate this point, the maximum and minimum densities yielded by the two wetting boundary conditions are compared in Table I for different contact angles. From the table we can see that the maximum and minimum densities given by the improved wetting boundary condition are in excellent agreement with the equilibrium liquid and gas densities $\left(\rho_{l} \approx 7.52\right.$ and $\left.\rho_{g} \approx 0.46\right)$, respectively. However, using the original wetting boundary condition [42], the maximum density considerably deviates from $\rho_{l}$ when $\theta<90^{\circ}$ and the minimum density deviates from $\rho_{g}$ when $\theta>90^{\circ}$, which means that the thermodynamic consistency of the free-energy LB model has been broken by the original wetting boundary condition [42].

Furthermore, the spurious velocities yielded by the improved wetting boundary condition are found to be significantly smaller than those given by the original wetting boundary condition [42]. The velocity fields obtained by the two wetting boundary conditions are compared in Fig. 4. Owing to the symmetry, only half of the computational domain is shown. From the figure it can be seen that, when the original wetting boundary condition [42] is used, the spurious velocities are very large near the three-phase contact lines (reduce to contact points in two dimensions). On the contrary, Fig. 4(b) shows that the spurious velocities yielded by the improved wetting boundary condition are very small. Quantitatively, the maximum spurious velocities given by the two wetting boundary conditions are compared in Fig. 5 for different cases. The figure shows that the maximum spurious velocities increase as the contact angle deviates from $\theta \approx 90^{\circ}$. Specifically, it can be found that the maximum spurious velocities caused by the improved wetting boundary condition are basically on the order of $10^{-5}$, which are smaller by three orders of magnitude than those given by the original wetting boundary condition [42]. Besides, the average deviation of the velocity field is also evaluated. For the cases of $\theta \approx 34^{\circ}, 61^{\circ}$, and $152^{\circ}$, the average deviations of the velocity fields yielded by the original

TABLE I. Comparison of the maximum and minimum densities produced by the original wetting boundary condition [42] and the improved wetting boundary condition for different contact angles.

\begin{tabular}{lcccr}
\hline \hline & \multicolumn{2}{c}{ Original wetting B.C. [42] } & \multicolumn{2}{c}{ Improved wetting B.C. } \\
\cline { 2 - 4 }$\theta$ (degrees) & $\rho_{\max }$ & $\rho_{\min }$ & $\rho_{\max }$ & $\rho_{\min }$ \\
\hline 34 & $8.038(6.9 \%)$ & $0.451(2.0 \%)$ & $7.499(0.3 \%)$ & $0.451(2.0 \%)$ \\
61 & $7.902(5.1 \%)$ & $0.456(0.9 \%)$ & $7.508(0.2 \%)$ & $0.456(0.9 \%)$ \\
90 & $7.568(0.6 \%)$ & $0.461(0.2 \%)$ & $7.519(0.0 \%)$ & $0.461(0.2 \%)$ \\
121 & $7.586(0.9 \%)$ & $0.430(6.5 \%)$ & $7.531(0.14 \%)$ & $0.465(1.1 \%)$ \\
152 & $7.599(1.1 \%)$ & $0.409(11.1 \%)$ & & $0.467(1.5 \%)$ \\
\hline \hline
\end{tabular}



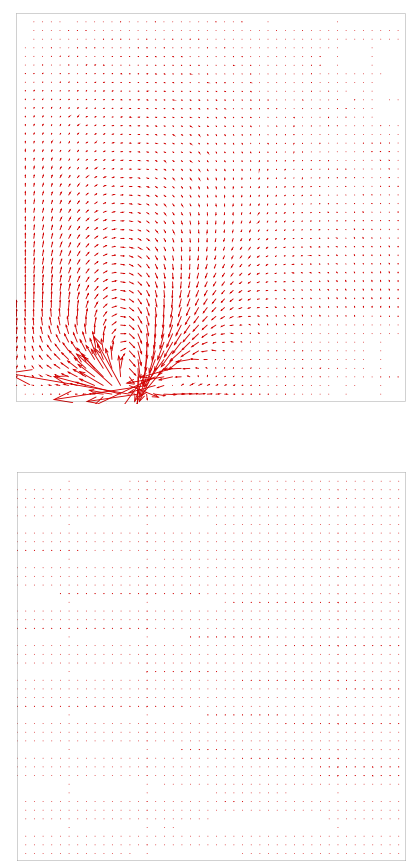

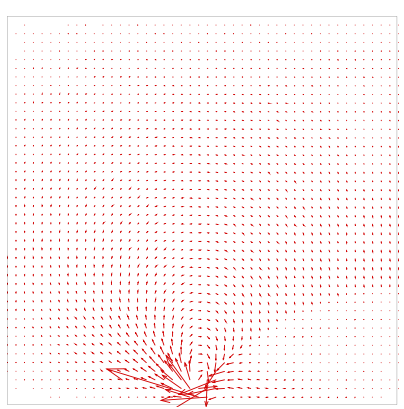

(a)

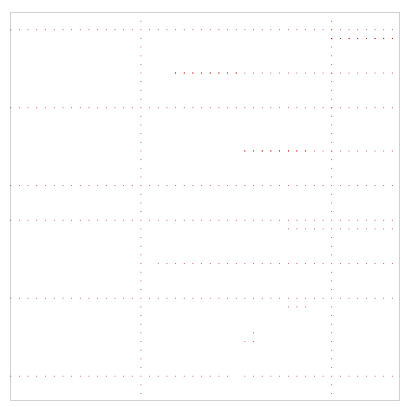

(b)
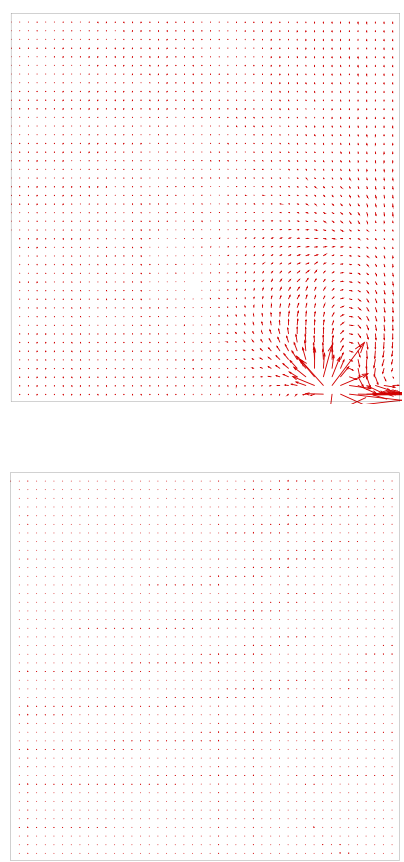

FIG. 4. The velocity fields obtained by (a) the original wetting boundary condition [42] and (b) the improved wetting boundary condition in the cases of (from left to right) $\theta \approx 34^{\circ}, 61^{\circ}$, and $152^{\circ}$, respectively. Owing to the symmetry, only half of the computational domain is shown.

wetting boundary condition are $1.5 \times 10^{-4}, 5.7 \times 10^{-5}$, and $9.3 \times 10^{-5}$, respectively, while the average deviations produced by the improved wetting boundary condition are $9.8 \times$ $10^{-7}, 2.5 \times 10^{-7}$, and $2.4 \times 10^{-6}$, respectively.

\section{B. Simulation of dynamic contact angles}

In this subsection, the capability of the proposed wetting boundary condition for simulating dynamic contact angles is numerically validated by testing the Cox-Voinov law [53-60]. We consider the movement of a liquid film between two infinite flat parallel plates. The computational domain is taken as

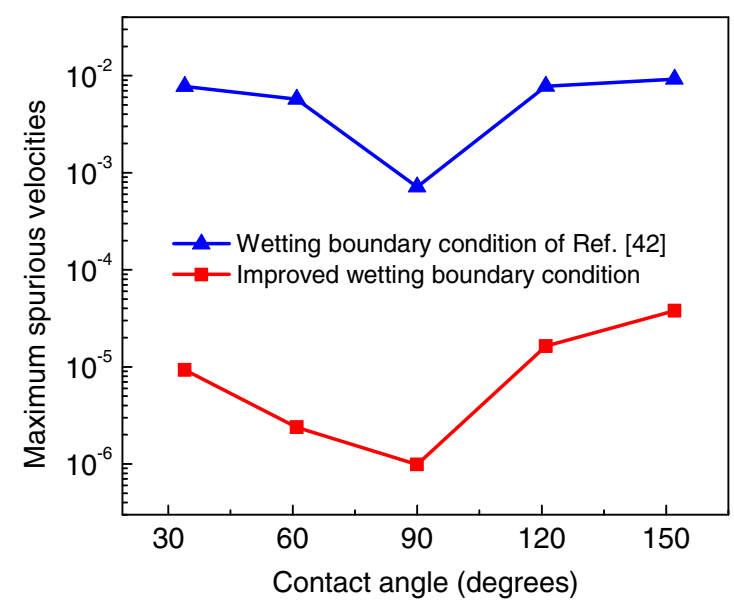

FIG. 5. Comparison of the maximum spurious velocities produced by the original wetting boundary condition [42] and the improved wetting boundary condition for different cases.
$L_{x} \times L_{y}=300$ 1.u. $\times 50$ 1.u. A liquid film is initially located at the center of the computational domain (see Fig. 6(a)) and its width is $601 . u$. The static contact angle of the two flat parallel plates is chosen as $\theta_{\mathrm{eq}} \approx 58.5^{\circ}$ and the kinematic viscosity is taken as $v=0.15$. To mimic the pressure-driven planar Poiseuille flow, a horizontal body force $\rho \mathbf{a}$ is applied to the computational domain with $\mathbf{a}=\left(a_{x}, 0\right)$ and the periodic boundary condition is employed in the horizontal direction. A steady state will be achieved in which the liquid film migrates along the channel at a constant speed [59], with the contact lines moving along each wall with a measured velocity $U_{\mathrm{CL}}$. The steady movement of the liquid film is expected to follow the Cox-Voinov law [53-57]:

$$
\left(\theta_{d}^{3}-\theta_{\text {eq }}^{3}\right) \propto \mathrm{Ca},
$$

where $\theta_{d}$ is the dynamic advancing contact angle and $\mathrm{Ca}=$ $\mu U_{\mathrm{CL}} / \sigma$ is the Capillary number, in which $\sigma$ is the surface

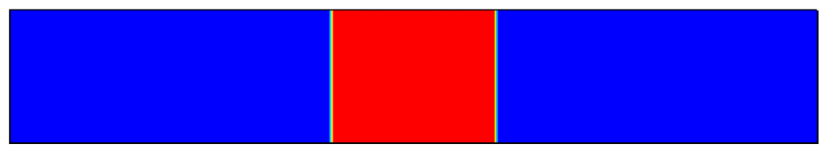

(a)

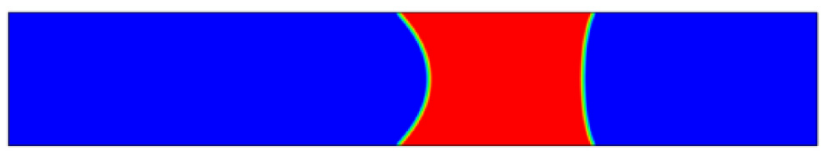

(b)

FIG. 6. Simulation of the movement of a liquid film between two infinite flat parallel plates. (a) The initial state and (b) a snapshot of the case $\mathrm{Ca} \approx 0.043$ at $t=4 \times 10^{4} \delta_{t}$. 


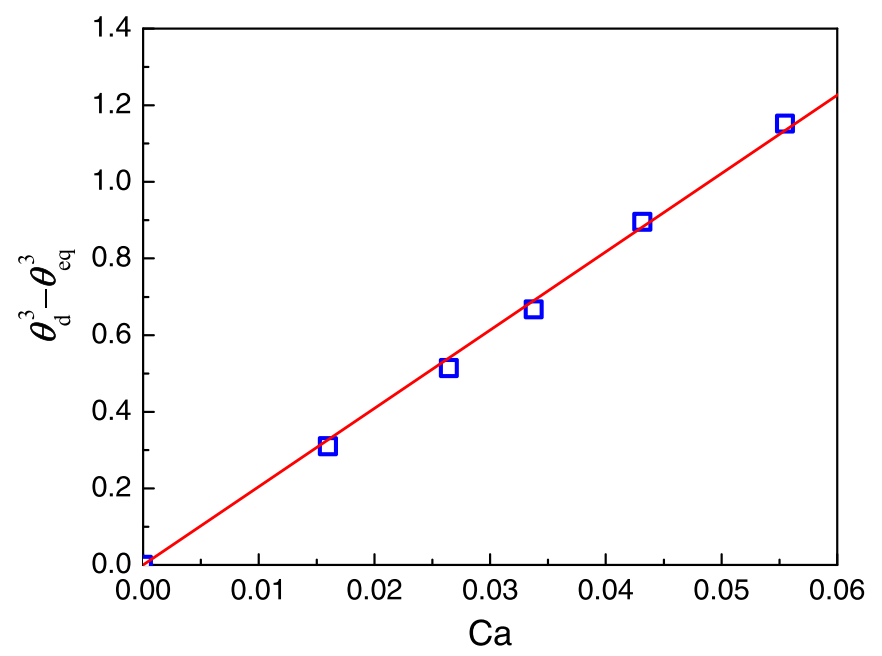

FIG. 7. Numerical validation of the Cox-Voinov law. The linear relationship between $\left(\theta_{d}^{3}-\theta_{\mathrm{eq}}^{3}\right)$ and the Capillary number. In this figure the contact angles $\theta_{d}$ and $\theta_{\mathrm{eq}}$ are expressed in terms of radians rather than degrees.

tension and $\mu=\rho_{l} v$ is the dynamic viscosity. Here it should be noted that Eq. (14) is valid for small Ca numbers [61-64]. Previous experimental studies have shown that the law given by Eq. (14) does not hold for $\mathrm{Ca}>0.1$ [65]. Besides, as mentioned by Göhl et al. [62] and Kovalev et al. [64], Eq. (14) can be applied on advancing contact lines, in which the contact angle increases with increasing contact line velocity. In the cases of receding contact angles, Eq. (14) no longer provides an adequate approximation [64] and readers are referred to some related studies listed in Ref. [62]. In our simulations, different cases are considered by varying $a_{x}$ from $0.375 \times$ $10^{-5}$ to $1.25 \times 10^{-5}$. Correspondingly, the Capillary number varies from $\mathrm{Ca} \approx 0.016$ to $\mathrm{Ca} \approx 0.056$. A snapshot of the case $\mathrm{Ca} \approx 0.043$ at $t=4 \times 10^{4} \delta_{t}$ is shown in Fig. 6(b). Our numerical results of $\left(\theta_{d}^{3}-\theta_{\mathrm{eq}}^{3}\right)$ are displayed in Fig. 7, which clearly verifies the linear relationship between $\left(\theta_{d}^{3}-\theta_{\text {eq }}^{3}\right)$ and the Capillary number.

\section{Droplet resting on a cylindrical surface}

Now we turn our attention to verifying the capability of the improved wetting boundary condition for modeling a droplet resting on a cylindrical surface with different contact angles. The computational domain is chosen as $L_{x} \times L_{y}=180$ 1.u. $\times$ 200 1.u. A solid circular cylinder of radius $R_{s}=401$.u. is located at $(90,100)$ and a droplet of the same radius is placed above the circular cylinder with its center at $(90,65)$.
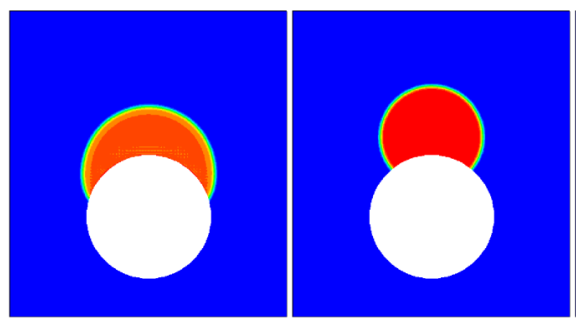

(a)
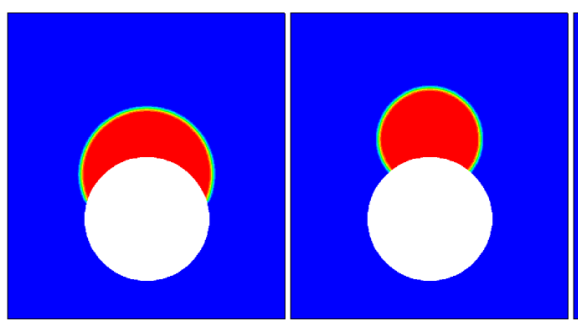

(b)
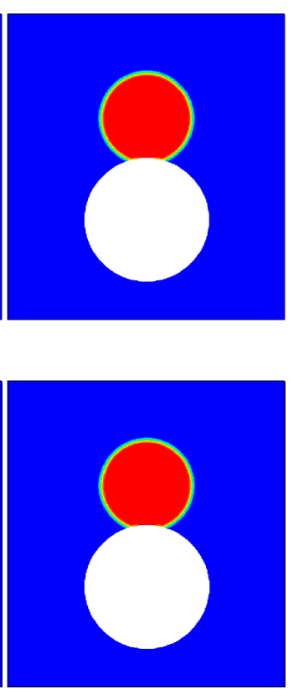

FIG. 8. Simulation of a droplet resting on a cylindrical surface. Comparison of the density contours obtained by (a) the original wetting boundary condition [42] and (b) the improved wetting boundary condition. From left to right: $\theta \approx 42^{\circ}, 90^{\circ}$, and $142^{\circ}$, respectively.

The periodic boundary condition is applied in the $x$ and $y$ directions, while the halfway bounce-back scheme and the wetting boundary condition are employed at the cylindrical surface. The parameters in Eq. (8) and the kinematic viscosity are the same as those used in the previous subsections. The temperature is still chosen as $T=0.7 T_{c}$, which corresponds to $\rho_{l} \approx 7.52$ and $\rho_{g} \approx 0.46$.

The density contours obtained by the original wetting boundary condition [42] and the improved wetting boundary condition at $t=4 \times 10^{4} \delta_{t}$ are displayed in Figs. 8(a) and 8(b), respectively, for different contact angles. The capability of the improved wetting boundary condition for simulating contact angles on a curved boundary is well verified. Particularly, by comparing the results of the two wetting boundary conditions in the case of $\theta \approx 42^{\circ}$, some obvious differences can be observed, which mainly arise from the fact that the thermodynamic consistency of the free-energy LB model is retained by the improved wetting boundary condition but is lost when employing the original wetting boundary condition [42]. The maximum and minimum densities yielded by the two wetting boundary conditions are compared in Table II. Similar to the test of contact angles on flat surfaces, the present test also shows that the maximum and minimum densities given by the improved wetting boundary condition agree very well

TABLE II. Comparison of the maximum and minimum densities produced by the original wetting boundary condition [42] and the improved wetting boundary condition for different contact angles on a cylindrical surface.

\begin{tabular}{lcccr}
\hline \hline & \multicolumn{2}{c}{ Original wetting B.C. [42] } & \multicolumn{2}{c}{ Improved wetting B.C. } \\
\cline { 2 - 4 }$\theta$ (degrees) & $\rho_{\max }$ & $\rho_{\min }$ & $\rho_{\max }$ & $\rho_{\min }$ \\
\hline 42 & $8.287(10.2 \%)$ & $0.455(0.9 \%)$ & $7.512(0.1 \%)$ & $0.458(0.43 \%)$ \\
90 & $7.518(0.03 \%)$ & $0.460(0.0 \%)$ & $7.517(0.04 \%)$ & $0.460(0.00 \%)$ \\
142 & $7.572(0.69 \%)$ & $0.395(14.1 \%)$ & $7.520(0.00 \%)$ & $0.462(0.43 \%)$ \\
\hline \hline
\end{tabular}




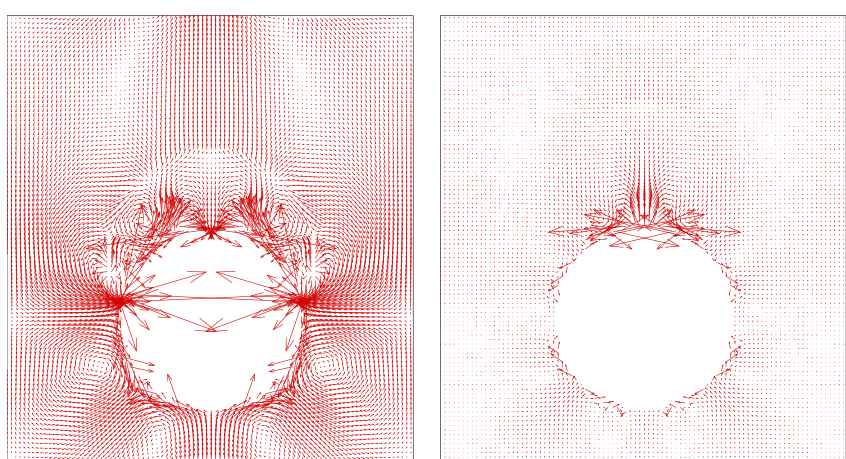

(a)

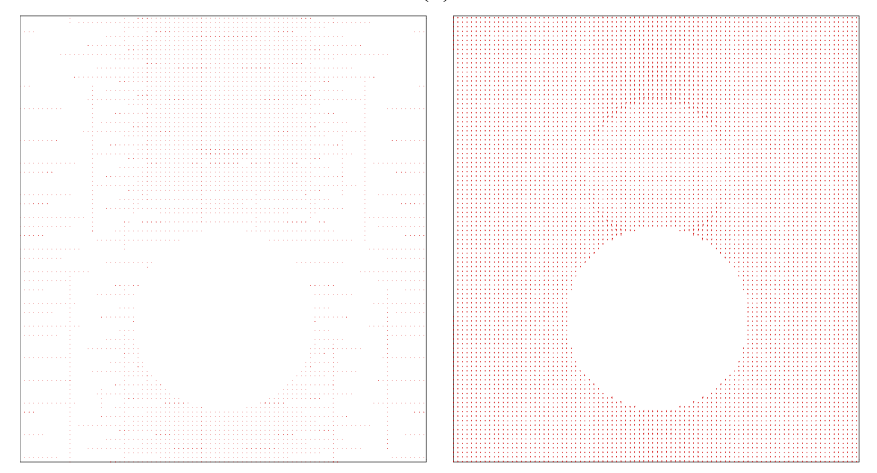

(b)

FIG. 9. The velocity fields obtained by (a) the original wetting boundary condition [42] and (b) the improved wetting boundary condition in the cases of (from left to right) $\theta \approx 42^{\circ}$ and $142^{\circ}$, respectively. For these two cases, the maximum spurious velocities caused by the original wetting boundary condition are $1.44 \times 10^{-2}$ and $1.23 \times 10^{-2}$, respectively, while the maximum spurious velocities yielded by the improved wetting boundary condition are $1.03 \times 10^{-5}$ and $1.49 \times 10^{-4}$, respectively.

with the equilibrium liquid and gas densities $\left(\rho_{l} \approx 7.52\right.$ and $\left.\rho_{g} \approx 0.46\right)$, respectively, while the maximum density yielded by the original wetting boundary condition [42] deviates from $\rho_{l}$ when $\theta<90^{\circ}$ and the minimum density deviates from $\rho_{g}$ when $\theta>90^{\circ}$.

The spurious velocities yielded by the two wetting boundary conditions are compared in Fig. 9 for cases of $\theta \approx 42^{\circ}$ and $142^{\circ}$. From the figure it can be clearly seen that the spurious velocities produced by the original wetting boundary condition [42] are much larger than those caused by the improved wetting boundary condition. Actually, for the original wetting boundary condition [42], the surface chemical potential will gradually deviate from the chemical potential of the fluid domain when $\theta>90^{\circ}$ or $\theta<90^{\circ}$, leading to a chemical potential gradient at the boundary node, which may be a source of the spurious velocities. For the cases of $\theta \approx 42^{\circ}$ and $142^{\circ}$, the average deviations of the velocity fields given by the original wetting boundary condition are $2.4 \times 10^{-4}$ and $7.0 \times 10^{-5}$, respectively, whereas the average deviations yielded by the improved wetting boundary condition are $2.2 \times 10^{-6}$ and $1.8 \times 10^{-5}$, respectively. Moreover, unlike Fig. 4(a), in which the large spurious velocities mainly appear around the three-phase contact lines, Fig. 9(a) shows that the original wetting boundary condition [42] leads to relatively large spurious velocities near the whole cylindrical surface. Such a phenomenon may be related to the curved boundary. However, it can be seen that the improved wetting boundary condition performs well for both straight and curved surfaces.

\section{CONCLUSIONS}

In this paper, we have proposed an improved wetting boundary condition to implement the wettability of a solid surface for the chemical-potential-based free-energy LB model. In comparison with the original wetting boundary condition [42], the improved wetting boundary condition has the following features. First, it employs a surface chemical potential that is compatible with the chemical potential of the fluid domain, while the surface chemical potential of the original wetting boundary condition is adjusted over a wide range to tune the contact angle, which may lead to a chemical potential gradient at each boundary node. Second, the numerical results show that the thermodynamic consistency of the free-energy LB model is basically retained by the improved wetting boundary condition but is lost when using the original wetting boundary condition. As a result, the maximum density yielded by the original wetting boundary condition considerably deviates from $\rho_{l}$ when $\theta<90^{\circ}$ and the minimum density deviates from $\rho_{g}$ when $\theta>90^{\circ}$. Furthermore, the maximum spurious velocities produced by the improved wetting boundary condition are found to be smaller by $2 \sim 3$ orders of magnitude than those yielded by the original wetting boundary condition. Finally, it is worth mentioning that the proposed wetting boundary condition can be directly extended to the 3D space.

\section{ACKNOWLEDGMENTS}

This work was supported by the National Natural Science Foundation of China (No. 51822606 and No. 52006244).
[1] R. Scardovelli and S. Zaleski, Annu. Rev. Fluid Mech. 31, 567 (1999).

[2] S. O. Unverdi and G. Tryggvason, J. Comput. Phys. 100, 25 (1992).

[3] C. W. Hirt and B. D. Nichols, J. Comput. Phys. 39, 201 (1981).

[4] J. A. Sethian and P. Smereka, Annu. Rev. Fluid Mech. 35, 341 (2003).
[5] H. Huang, J.-J. Huang, and X.-Y. Lu, Comput. Fluids 93, 164 (2014).

[6] S. Chen and G. D. Doolen, Annu. Rev. Fluid Mech. 30, 329 (1998).

[7] C. K. Aidun and J. R. Clausen, Annu. Rev. Fluid Mech. 42, 439 (2010)

[8] S. Succi, Europhys. Lett. 109, 50001 (2015). 
[9] Z. Guo and C. Shu, Lattice Boltzmann Method and Its Applications in Engineering (World Scientific, Singapore, 2013).

[10] Q. Li, K. H. Luo, Q. J. Kang, Y. L. He, Q. Chen, and Q. Liu, Prog. Energy Combust. Sci. 52, 62 (2016).

[11] Q. Li, Y. Yu, and Z. X.Wen, Phys. Fluids 32, 093306 (2020).

[12] X. Shan and H. Chen, Phys. Rev. E 47, 1815 (1993).

[13] X. Shan and H. Chen, Phys. Rev. E 49, 2941 (1994).

[14] X. Shan, Phys. Rev. E 77, 066702 (2008).

[15] D. Zhang, K. Papadikis, and S. Gu, Int. J. Multiphase Flow 64, 11 (2014).

[16] Q. Li, K. H. Luo, and X. J. Li, Phys. Rev. E 87, 053301 (2013).

[17] A. Xu, T. S. Zhao, L. An, and L. Shi, Int. J. Heat Fluid Flow 56, 261 (2015).

[18] Q. Li, D. H. Du, L. L. Fei, and K. H. Luo, Comput. Fluids 186, 128 (2019).

[19] X. Shan, Phys. Rev. E 73, 047701 (2006).

[20] Q. Li, K. H. Luo, and X. J. Li, Phys. Rev. E 86, 016709 (2012).

[21] A. K. Gunstensen, D. H. Rothman, S. Zaleski, and G. Zanetti, Phys. Rev. A 43, 4320 (1991).

[22] D. Grunau, S. Chen, and K. Eggert, Phys. Fluids A 5, 2557 (1993).

[23] H. Liu, A. J. Valocchi, and Q. Kang, Phys. Rev. E 85, 046309 (2012).

[24] M. R. Swift, W. R. Osborn, and J. M. Yeomans, Phys. Rev. Lett. 75, 830 (1995).

[25] M. R. Swift, E. Orlandini, W. R. Osborn, and J. M. Yeomans, Phys. Rev. E 54, 5041 (1996).

[26] X. He, S. Chen, and R. Zhang, J. Comput. Phys. 152, 642 (1999).

[27] T. Lee and C.-L. Lin, J. Comput. Phys. 206, 16 (2005).

[28] Q. Li, K. H. Luo, Y. J. Gao, and Y. L. He, Phys. Rev. E 85, 026704 (2012).

[29] T. Krüger, H. Kusumaatmaja, A. Kuzmin, O. Shardt, G. Silva, and E. M. Viggen, The Lattice Boltzmann Method - Principles and Practice (Springer Nature, Berlin, 2017).

[30] A. Kuzmin, A. A. Mohamad, and S. Succi, Inter. J. Mod. Phys. C 19, 875 (2008).

[31] T. Inamuro, N. Konishi, and F. Ogino, Comput. Phys. Commun. 129, 32 (2000).

[32] A. N. Kalarakis, V. N. Burganos, and A. C. Payatakes, Phys. Rev. E 65, 056702 (2002).

[33] C. M. Pooley and K. Furtado, Phys. Rev. E 77, 046702 (2008).

[34] A. J. Wagner and Q. Li, Physica A 362, 105 (2006).

[35] T. Lee and P. F. Fischer, Phys. Rev. E 74, 046709 (2006).

[36] A. Mazloomi M, S. S. Chikatamarla, and I. V. Karlin, Phys. Rev. Lett. 114, 174502 (2015).
[37] B. Wen, L. Zhao, W. Qiu, Y. Ye, and X. Shan, Phys. Rev. E 102, 013303 (2020).

[38] A. J. Briant, A. J. Wagner, and J. M. Yeomans, Phys. Rev. E 69, 031602 (2004).

[39] A. Dupuis and J. M. Yeomans, Future Gener. Comput. Syst. 20, 993 (2004).

[40] P. Yuan and L. Schaefer, Phys. Fluids 18, 042101 (2006).

[41] A. Mazloomi M., S. S. Chikatamarla, and I. V. Karlin, Phys. Rev. E 92, 023308 (2015).

[42] B. Wen, X. Zhou, B. He, C. Zhang, and H. Fang, Phys. Rev. E 95, 063305 (2017).

[43] P. Lallemand and L.-S. Luo, Phys. Rev. E 61, 6546 (2000).

[44] L.-S. Luo, W. Liao, X. Chen, Y. Peng, and W. Zhang, Phys. Rev. E 83, 056710 (2011).

[45] M. E. McCracken and J. Abraham, Phys. Rev. E 71, 036701 (2005).

[46] K. N. Premnath and J. Abraham, J. Comput. Phys. 224, 539 (2007).

[47] Z. Guo, C. Zheng, and B. Shi, Phys. Rev. E 83, 036707 (2011).

[48] Q. Lou and Z. Guo, Phys. Rev. E 91, 013302 (2015).

[49] Q. Li, Y. Yu, and R. Z. Huang, Phys. Rev. E 103, 013304 (2021).

[50] Q. Li, Y. Yu, and K. H. Luo, Phys. Rev. E 100, 053313 (2019).

[51] H. Ding and P. D. M. Spelt, Phys. Rev. E 75, 046708 (2007).

[52] H.-R. Liu and H. Ding, J. Comput. Phys. 294, 484 (2015).

[53] O. V. Voinov, Fluid Dyn. 11, 714 (1976).

[54] R. G. Cox, J. Fluid Mech. 168, 169 (1986).

[55] P. Gao and X. Y. Lu, J. Fluid Mech. 724, R1 (2013).

[56] R. G. M. van der Sman, Comput. Phys. Commun. 184, 2751 (2013).

[57] J. Grawitter and H. Stark, Soft Matter 17, 2454 (2021).

[58] C. Monteux, Y. Elmaallem, T. Narita, and F. Lequeux, EPL 83, 34005 (2008).

[59] H. Kusumaatmaja, E. J. Hemingway, and S. M. Fielding, J. Fluid Mech. 788, 209 (2016).

[60] M. Latva-Kokko and D. H. Rothman, Phys. Rev. Lett. 98, 254503 (2007).

[61] Š. Šikalo, H.-D. Wilhelm, I. V. Roisman, S. Jakirlić, and C. Tropea, Phys. Fluids 17, 062103 (2005).

[62] J. Göhl, A. Mark, S. Sasic, and F. Edelvik, Int. J. Multiphase Flow 109, 164 (2018).

[63] S. Zitz, A. Scagliarini, S. Maddu, A. A. Darhuber, and J. Harting, Phys. Rev. E 100, 033313 (2019).

[64] A. V. Kovalev, A. A. Yagodnitsyna, and A. V. Bilsky, J. Phys. Conf. Ser. 1677, 012064 (2020).

[65] X. Wang, X. Peng, Y. Duan, and B. Wang, Chin. J. Chem. Eng. 15, 730 (2007). 